\title{
A concise review on lipidomics analysis in biological samples
}

\author{
Ramani Venkata Addepalli ${ }^{1}$, Ramesh Mullangi ${ }^{2 *}$ \\ ${ }^{1}$ Palm Meadows, Kompally, Hyderabad-500100, India \\ ${ }^{2}$ Laxai Life Sciences Pvt Ltd, MN Park, Genome Valley, Shamirpet, Hyderabad-500 078, India \\ *Corresponding Author: E-mail: ramesh.mullangi@laxai.com; Tel.: +91-9611333488; Fax: +91-40-66799998. \\ Received: October 09, 2020; Revised: November 23, 2020; Published: December 09, 2020
}

\begin{abstract}
Lipids are a complex and critical heterogeneous molecular entity, playing an intricate and key role in understanding biological activities and disease processes. Lipidomics aims to quantitatively define the lipid classes, including their molecular species. The analysis of the biological tissues and fluids are challenging due to the extreme sample complexity and occurrence of the molecular species as isomers or isobars. This review documents the overview of lipidomics workflow, beginning from the approaches of sample preparation, various analytical techniques and emphasizing the state-of-the-art mass spectrometry either by shotgun or coupled with liquid chromatography. We have considered the latest ion mobility spectroscopy technologies to deal with the vast number of structural isomers, different imaging techniques. All these techniques have their pitfalls and we have discussed how to circumvent them after reviewing the power of each technique with examples..
\end{abstract}

C2021 by the authors. This article is an open-access article distributed under the terms and conditions of the Creative Commons Attribution license (http://creativecommons.org/licenses/by/4.0/).

\section{Keywords}

Lipids; LC-MS/MS; samples processing; bioanalysis

\section{Introduction}

Lipids are a complex and critical heterogeneous molecular entity, playing an intricate and key role in many biological functions such as acting as structural scaffold for the cell membrane, serving as energy storage and participating in signaling pathways. Lipid classification system is spearheaded by LIPID MAPS ${ }^{\circledR}$ Lipidomics Gateway (https://www.lipidmaps.org/). Lipids can be divided into eight main categories: fatty acyls (FA), glycerolipids (GL), glycerophospholipids (GP), sphingolipids (SP), sterol lipids (ST), prenol lipids (PR), saccharolipids (SL) and polyketides (PK) [1] (Table 1). Each category can be further classified into different lipid classes and subclasses, based on the number of carbon atoms and double bonds, the branching of the hydrocarbon chain, the position and orientation of double bonds, the addition of polar groups such as choline, inositol and ethanolamine; and glycosylation [2]. The LIPID MAPS structure database currently records 45245 lipid structures (as of 12 March 2020) (https://www.lipidmaps.org/). The complete lipid profile within a cell, tissue or organism is known as a lipidome and is a branch of the "metabolome". Lipidomics is a discipline that studies the characteristics of lipid and to unravel the complex interactions of lipid metabolites in a biological system. 
Table 1. Examples of eight categories of lipids

Categories Structures Examples

Fatty acyls, FA<smiles>CCCCCCCCCCCCCCCC(=O)O</smiles>

Hexadecanoic acid

Prenol lipids, PR<smiles>CCCCCCCC(C)=CCCC(C)=CCO</smiles>
2E,6E-Farnesol

Glycerolipids, GL<smiles>CCCCCCCC/C=C\CCCCCCCCCCCCCC(=O)OCCCO</smiles>

1-Hexadecanoyl-2-(9Z-octadecenoyl)-sn-glycero

Glycerophospholipids,

GP<smiles>CCCCCCCCCCCCCCCCCCCCCCCC(=O)OC[C@H](OCC(=O)CCCCCCCCCC)OP(=O)(O)OC[NH2+]C</smiles>

1-Hexadecanoyl-2-(9Z-octadecenoyl)-sn-glycero-3phosphocholine

Sphingolipids, SP<smiles>CCCCCCCC=C[C@@H](O)[C@H](CO)NC(=O)CCCCCCCCCCCCCCCCCCCCC</smiles>
$\mathrm{N}$-(Tetradecanoyl)-sphing-4-enine

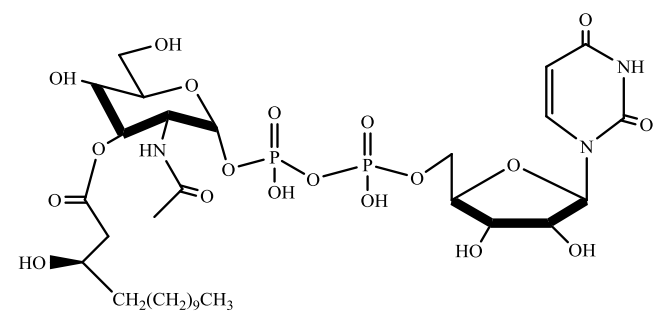

UDP-3-O-(3R-Hydroxyl-tetradecanoyl)- $\alpha \mathrm{D}$-N-acetylglucosamine

Sterol lipids,

ST

Polyketides,

PK<smiles>CCCCC(C)C1CCC2[C@H]3CC=C4C[C@@H](O)CC[C@]4(C)[C@H]3CC[C@]12C</smiles>

Cholest-5-en-3ß-ol<smiles>COc1cc2c(c3oc(=O)c4c(c13)CCC4=O)[C@H]1C=CO[C@@H]1O2</smiles>

Sterols

Cholesterol and derivatives Steroids

Bile acids and derivatives

Macrolide polyketides Aromatic polyketides

Nonribosomal peptide/ polyketides hybrids

A specific alteration in the lipidome, provides potential insights into perturbed pathways, physiological processes and ultimately stages of diseases. Clinical lipidomics was defined "as a new integrative 
biomedicine to discover the correlation and regulation between a large scale of lipid elements measured and analyzed in liquid biopsies from patients with those patient phenomes and clinical phenotypes" [3]. To cite few examples, lysophosphatidic acid stimulates cell proliferation, migration and survival by acting on its cognate G-protein-coupled receptors in cancer cells [4]. Alzheimer's and Parkinson's diseases have been linked with aberrant cholesterol and abnormal glycolipid metabolism, respectively [5]. Lipoprotein abnormalities are associated with Type II diabetes with increased triglycerides (TG) and very low density lipoprotein (VLDL) levels, whereas decreased apolipoprotein E/VLDL-TG ratio in ischemic heart disease [6]. Depending on associated underlying pathways, these lipids serve as a potential biomarkers and a diagnostic tool. For instance, GL, SP, linoleic acid, cholesterol serve as a biomarker for Alzheimer's; lycophosphatidylinositol and prostaglandins (PG) for Parkinson's; ceramides, sphingomyelin for Type II diabetes mellitus and glycosphingolipid for obesity [7].

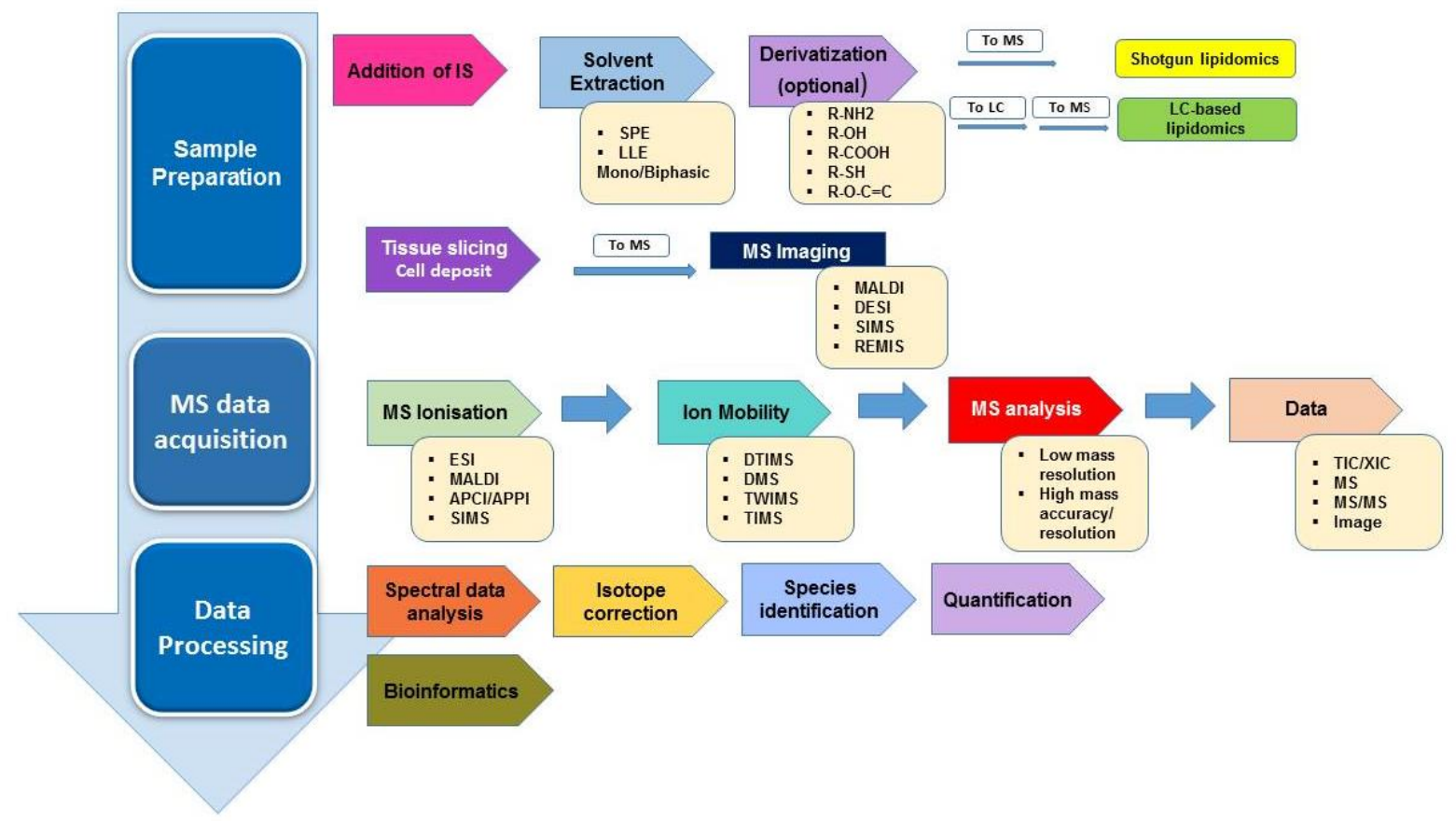

Figure 1. Typical work-flow of lipidomics analysis in biological samples

Owing to the diversification of the lipid classes with various combinations of polar head groups, fatty acyl chains, backbone structures, identification and characterization of lipids is very complicated. Aggravating to this is its extensive expansion of the applications. The prime objective of lipidomics is to attain full coverage of precise structural analysis, accurate quantification and understanding its dynamics. The available analytical techniques are broadly categorized into three groups, namely global lipidomic analysis, targeted lipidomic analysis and novel lipid discovery. The global lipidomics deals with identification, quantification hundreds to thousands of cellular lipids via a high throughput basis. Shotgun lipidomics-based platforms are extensively used in this category to analyze diverse pathways and networks associated with lipid metabolism, trafficking, and homeostasis. As an extension, mapping techniques have also been used to study the spatial and temporal relationships of lipids. Targeted lipidomic analysis also deals with the identification but with one or a few lipid classes of interest using LC-MS (liquid chromatography coupled to mass spectrometry) and LC-MS/MS (liquid chromatography coupled to tandem mass spectrometry) based methods. While the novel lipid discovery deal with novel lipid classes and molecular species using LC coupled with MS with different enrichment technologies. In this review, we are providing an overview of the current understanding of lipid analysis taking into account the workflow, 
methodologies, technical characteristics and bottlenecks. We have also listed important papers and reviews that cover most of the aspects of lipidomics. We hope that this review will act as a bridge for biomedical and pharmacological research to generate novel approaches to disease diagnostics. A typical work flow of lipidomic analysis in biological samples is as shown Figure 1.

\section{Sample preparation}

\section{Sample processing and storage}

The most important and vital step in any bioanalytical work is sample processing. The biological samples can be solid in nature (e.g., tissues or cells) or comprise of highly complex biofluids (e.g., plasma, serum, urine or cyst fluid). It is laborious to handle solid samples as it involves additional disruption step. It is highly advisable to process the samples immediately, especially with the whole blood samples [8] or at least flash frozen as lipid samples exhibit substantial circadian variations. It is a well-known fact that the plasma concentration of lysophosphatidylcholine (LPC) or lysophosphatidic acid (LPA) increases when left at room temperature for a long period [9]. On the other hand, cardiolipin (CL) during the freezing process hydrolyses into monolysocardiolipin [10] and methanolic samples of lysophospholipid regioisomers isomerize at temperatures above $20^{\circ} \mathrm{C}$ and at $\mathrm{pH}>6.0$ [11]. Freeze-thaw is another problem with specific lipid classes, aliquoting the samples before freezing will minimize its effect, example sphingosine, polyunsaturated fatty acids and eicosanoids [12,13]. Yet another problem is lipid oxidation and is a major concern with polyunsaturated fatty acid moieties, oxidized lipids and eicosanoids, special care should be exercised in handling, as these end products also contribute to both physiological and pathophysiological processes [13]. Storage of plasma/serum samples and their extracts under an inert gas (e.g., argon) may also limit oxidation. Antioxidants like butylhydroxytoluene (BHT) have been in use, the used concentrations and the time-point of its addition vary in the literature [14]. The efficacy and protocols for the use of antioxidants should be verified. However, oxidation is not a problem in quantifying abundant lipid classes (e.g., phospholipids, sphingolipids and TGs) [13]. It is advisable to exercise caution while handling samples with each class of lipids.

Biofluids, such as urine, serum, plasma and whole blood are frequently used as these do not require any homogenization but while working with the lipids from a piece of tissue or ruptured cells (e.g., organelles), sample disruption nonetheless has a significant impact on the end results of a process to make it accessible to extraction solvents. Widely used mechanical methods are liquid based homogenization (Potter-Elvehjem homogenizer, ULTRA-TURRAX), bead bearing (Bead Ruptor 24, Elite Bead Mill Homogenizer) and crushing of liquid-nitrogen-frozen tissue by pestle and mortar [15]. Latter approach is very slow as it is performed manually. Ulmer et al. (2017) have demonstrated the use of zirconium oxide or ceramic bead for softer tissues and stainless steel bead for muscle and harder tissues [16].

\section{Lipid extraction protocols}

There are numerous published lipid extraction methods that can also be automated for high-throughput analysis [17-19]. This process reduces the complexity of the sample by enriching the analytes of interest and getting rid of any unwanted contaminants to mass spectrometer. The extraction principles are one/two-phase liquid-liquid extraction (LLE), solid-phase extraction (SPE) with varying parameters of extraction like temperatures, sample/solvent ratio, re-extractions, use of sonication, vortexing, extraction under inert gasses to enhance the lipid recovery. However, this process is accountable for the artifacts in lipid identification and inconsistencies in quantification. 
The sample preparation technique most widely used in lipidomics is LLE. The Folch protocol and the Bligh and Dyer protocol both rely on a ternary mixture of chloroform, methanol and water $[17,18]$. Onephase extraction (OPE) method was proposed by Pellegrino et al. (2014) which is a mixture of methanol/chloroform/methyl tert-butyl ether (MMC) in the ratio 1.33/1/1 (v/v/v) [20]. Matyash et al. (2008) and Baker et al. (2001) proposed methyl-tert-butyl ether high-throughput lipidomics and acidified butanol extraction procedure for lysophosphatidic acid from biological samples [19,21]. It is well known fact that solubility can be enhanced by addition of some acid to the organic layer containing anionic lipids e.g. phosphatidic acids (PA), phosphatidylinositols (PI) or sphingosine-1-phosphate (S1P) to increase the extraction efficiency. We recommend to check the website http://cyberlipid.gerli.com/techniques-ofanalysis/ extraction-handling-of-extracts/ for guidance on extraction protocols.

\section{Derivatization}

Chemical derivatization has substantially improved the shortcomings of mass spectrometric based shotgun lipidomics, liquid chromatography and more so in gas chromatography based applications. Though it is an additional step, it offers several advantages, it enhances the ionization efficiency, selectively introduce a fragment which can be used in precursor ion or neutral loss scans, masks the functional groups that contaminate the mass spectrometer (MS), often encountered with the lipids containing phosphates and most importantly, it helps in differential quantitation by selectively introducing an isotopic label. All these strategies were used by different scientists. The plasma samples for 7-oxocholesterol and 5,6epoxycholesterol were chemically derivatized with Girard's reagent $P$ to increase the ionization efficiency of the intermediate metabolites in the patients suffering from lysosomal storage disorders [22]. Wang et al. (2017) quantitatively analyzed phosphatidylglycerols and bis(monoacylglycero) phosphates by diazomethane-based methylation of phosphate group to introduces class-specific fragments into the MS/MS spectra [23]. To prevent the contamination of the MS, Clark et al. (2011) methylated the phosphate groups to quantify phosphatidyl inositol phosphates [24]. Last strategy was adopted by Lee et al. (2017) who introduced a stable isotope-labeled methylation into one sample to enhanced detection and quantification of targeted phospholipids [25]. Chemical derivatization with $\mathrm{N}$-[4-(amino methyl) phenyl] pyridinium prevented the molecular masses overlapping signals between ceramides and branched fatty acid esters of hydroxy fatty acids (FAHFAs) to a new region thereby reducing the false results of FAHFAs [26]. New chemical derivatization approaches were employed on targeted lipids by Zhao et al. (2020) [27]. However, there are lot of shortcomings in derivatization procedures and such procedures are expected to be rapid, high yielding, specific, in-situ, or biocompatible in order to meet the needs of studies.

\section{Internal standards}

Internal standards are required to quantify bonafide concentrations of an analyte of interest in MS analysis. Both the internal standard and analyte are analyzed simultaneously to compensate for inherent variations in sample processing during the entire process of sample preparation and analysis (e.g., variations in lipid extraction efficacy, processing losses, matrix effects i.e., ionization suppression or enhancement). In an ideal scenario, both the internal standard and the analyte should be structurally similar and have a comparable MS/MS fragmentation pattern. So, a clear understanding of the types, concentration and characteristics of internal standards to be used for accurate quantification of lipid species, subclasses, and classes is of utmost importance. Ideally a stable isotope of the analyte of interest, if commercially available, should be used as an internal standard for quantitative analysis, provided quantification is limited to one or a limited number of species $[28,29]$. 
Internal standard mixes in lipidomic analysis approach is very common to biomarker discovery. Few researchers are of the opinion that one internal standard per lipid class is enough for quantitation [29], because ionization of lipids is largely dependent on the class specific head group and not so much on the fatty acyl chains [30]. Whereas, few others have contradictory report about the influence of fatty acyl chain length and unsaturation on ionization efficiency [30], it is highly advisable to extensively evaluate on case to case basis on this issue. The commercially available internal standard mixes containing isotope-labeled species include a wide range of acyl chain length and degree of saturation [31]. At least two internal standards are required to correct the effects of differential fragmentation kinetics and thermodynamics [32]. However, very few reliable internal standards with adequate chemical purity and exactly known lipid content are commercially available for a limited number of lipid subclasses and fatty acid compositions.

An internal standard cocktail has been developed for the LIPID MAPS Consortium which is commercially available as SPLASH ${ }^{\circ}$ LIPIDOMIX from Avanti Polar Lipids for quantitative mass spectrometry analysis (https://avantilipids.com/product/330707, 2018). This is specifically designed to complement human plasma lipid analysis using LC-MS/MS platforms. This contains uncommon chain-length sphingoid bases (C17) for sphingosine (So), sphinganine (Sa) and their 1-phosphates (S1P and Sa1P) and C12:0 fatty acid analogs of Ceramide (Cer) ceramide 1-phosphate (Cer1P), sphingomyelins (SM), simple mono- and dihexosylceramides (HexCer and diHexCer). It also includes sulfatides, 1-deoxy- and 1-(deoxymethyl)sphingoids, glycerol-phospholipids, phosphatidylinositol-bisphosphate, sterols and neutral lipids (https://avantilipids.com/product/330707, 2018).

To get consistent and robust results in a high throughput clinical analysis, the use of commercially available ready-made internal standard mixes with exactly known concentrations has an added advantage. The lipidomics community should encourage the development of novel comprehensive and easily available isotope labeled internal standard mixes.

\section{Analytical techniques for the study of lipids}

\section{Shotgun Lipidomics}

Identification and quantification of a cellular lipidomics directly from organic extracts of biological samples based on chemical and physical properties of lipid classes, subclasses, and individual molecular species are the prime aim of shotgun lipidomics [33]. This concept was based on a simple technique of a conventional loop injection, using a syringe coupled with tandem quadrupole mass spectrometry analysis of precursor masses and fragment mass, refraining from chromatographic separation [33,34]. This evolved into static nano-ESI source [29] without syringe pump; resulting in higher ionization efficiencies. Han et al. had used intra-source separation, favoring the ionization (i.e., positive- and negative-ion ionization) of selected lipid classes through solvent additives and subsequently using precursor ion and neutral loss scans of polar head group, resulting in fatty acid moieties [34,35]. Sufficient quantitation was achieved by addition of one internal standard per lipid class [36] as ionization largely dependents on specific head group and not so much on the fatty acyl chains [37]. Though some scientists contradict that fatty acyl chain length and unsaturation influence ionization efficiency [38]. Another direct infusion methodology adopted by Guan et al. (2006) was coupling triple quadrupole analyzer in multiple reactions monitoring (MRM) mode with syringe pump [39]. This was used to quantify the major lipid components in the lipid extract but critically lies in the knowledge of anticipated precursor and product ions. Although this technique is simple, ease of management and less expensive, due to the continuous infusion cross-contamination, isobaric overlaps of the $\mathrm{M}+2$ isotope with the monoisotopic peak of the compound are major limitations. 
To overcome the contamination problem from the carryover of previous samples, multi-dimensional mass spectrometry based shotgun lipidomics (MDMS-SL) was used to study the composition of lipid structures [37]. Using this technique differential intra-source separation properties with various additives like $\mathrm{Li}^{+}$or $\mathrm{NH}_{4}^{+}$or $\mathrm{Na}^{+}$, resulted in unique fragments for each lipid class. Nanoelectrospray ionization was integrated with chip-based nano-ESI platform (Advion NanoMat ${ }^{\circledR}$ ) for better sensitivity and high reproducibility of sample infusion. Quantitative applications of various classes of glycerophospholipids, sphingolipids and glycerolipids were studied using this technique. However, this devise is not cost effective and has limited stability of the electrospray due to clogging. Moreover, samples were subjected to analysis sequentially, by charge separation (e.g., deprotonation) or adduct formation (e.g., protonation, alkaline metal adduct ion, halogen adduct ion) making the data handling difficult where specialized software packages are required, which will be discussed later in this article. Other disadvantage being separation of isobaric lipids is not possible. In an unique application, differential mobility spectrometry (DMS) separation was combined with ionization by electrospray ionization (ESI) to separate many isobaric and isomeric lipids, which is also not fully evolved [40]. Nonetheless, this was a quick and reliable technique. Detection of unexpected lipid species and vulnerability for overlapping isobaric compounds is the major limitation of this method.

Automation was brought about by replacing syringe pump with HPLC (high-performance liquid chromatography) and coupling with triple quadrupole analyzer. The HPLC pump runs at micro-flow rates and the auto-sampler injects samples into the flow directly delivering into the ESI (electro-spray ionization) source sans the column [41]. Robustness and high automation made data acquisition simpler, as multiple precursor ions and constant neutral loss scans could be achieved by concentrated sample pulse and multiple injections. Multiple standard curves were achieved in quantitation of lipids (different fatty acyl chain lengths and degrees of unsaturation) by standard addition method and one internal standard [42]. This method was further applied on various subclasses of glycerophospholipids, sphingolipids and sterols [42]. However, low resolution direct infusion technologies suffer from general dogmas of limitations, such as ion suppression, ambiguous identification of isobaric/isomeric lipid species, and ion source-generated artifacts, hindering the applications to low-abundance lipid species, particularly in less ionizable or isomers bearing identical fragmentation patterns. One such example, diacyl and acyl-alkyl glycerophospholipids, isobaric phosphatidylcholines with odd-carbon-numbered fatty acids from plasmalogens [43].

To deal with this, shotgun lipidomics has evolved into a myriad of multi-dimensional strategies for molecular lipid characterization by coupling with high resolution mass spectrometers (HRMS) like quadrupole time of flight (Q-ToF) or Orbitrap or Fourier transform ion cyclotron resonance (FT-ICR) [44-46]. HRMS measures exact mass, time-of-flight MS (ToF-MS), Orbitrap MS and FT-ICR-MS deliver mass resolutions of $60,000,240,000$ and more than 1,000,000, respectively. HRMS is especially useful because of their rapid acquisition of MS/MS spectra, higher mass resolution and optional $\mathrm{MS}^{\mathrm{n}}$ fragmentation, enabling fingerprint studies without prior separation and eliminating the possibilities of false-positive identification. Choi et al. (2014) used untargeted lipid analysis to achieve detection of nine lipids in plasma after rosuvastatin treatment to explicate the side effects of the drug by using QToF-MS [46]. Further, this facilitates data-independent acquisition (DIA) MS/MS ${ }^{\mathrm{ALL}}$, which has a wide mass range of spectral coverage to perform qualitative analysis. Gao et al. were pragmatic in quantification of cardiolipin in mitochondrial preparations [47]. Major shortcoming of $\mathrm{MS} / \mathrm{MS}^{\mathrm{ALL}}$ is that it leads to eventual loss of information on precursor-fragment relationships, complicating the identification of lipids. Almeida et al. (2014) utilized full fragmentation power of an Orbitrap Fusion Tribrid and sequentially acquired higher-energy collisional induced dissociation (HCD) and collisional induced dissociation (CID) and ion trap mass spectrometry 
(ITMS3) to structurally characterize molecular glycerophospholipid species [44]. Using this novel high confidence filtering strategy, 311 lipid species circumventing 20 lipid classes and identification of 202 distinct molecular glycerophospholipid species in mouse cerebellum and hippocampus was achieved. Flaherty et al. (2019) evaluated the levels of multiple lipid species in bone marrow-derived macrophages using triple quadrupole/ion trap mass spectrometer [48].

\section{Liquid-chromatography-mass spectrometry}

Shotgun approach is extensively used because of its simple, rapid and ease of handling the crude lipid extracts. But the major pitfall of shotgun is highly convoluted spectra due to matrix interference (ion suppression and ion enhancement effects), functional group modification, and occurrence of the molecular species as isomers or isobars throws greatest challenges to separation scientists. This requires good analytical separation platforms i.e., LC-MS to reduce the above drawbacks. Further, coelution of lipids had replaced conventional HPLC systems with faster run times, highly efficient separation owing to the higher backpressure allowance, ultra-high-pressure liquid chromatography (UHPLC). Integrating UHPLC with ToFMS (UHPLC/Q-ToF-MS) is powerful tool, which enables high resolution chromatographic separation coupled with structure elucidation and identification of fragmentation patterns of the comprehensive nontargeted lipid analysis [49].

The most important separation techniques used in lipidomics are RPLC (reverse phased liquid chromatography), NPLC (normal phase liquid chromatography) and HILIC (hydrophilic interaction liquid chromatography). Other separation techniques are also occasionally used include non-aqueous RPLC (NARP) [50], silver-ion RPLC [51], chiral LC [52] and supercritical fluid chromatography (SFC) [53]. Each technique was used by different group of scientists to resolve the complexity of the lipidome. Firstly, nonaqueous RPLC was use by Lin et al. (1997) the separation of molecular species of 45 synthetic triacylglycerols and diacylglycerols, due to the advent of new column technologies this use is limited [50]. Secondly, non-aqueous reversed-phase (NARP) and silver-ion high-performance liquid chromatography with APCI-MS and GC/FID detection were used for the characterization of fatty acids and triglycerides composition in complex samples of animal fats [51]. Thirdly, complex mixtures of regioisomeric and enantiomeric eicosanoids (hydroxy and hydroperoxy fatty acids) have been resolved using chiral lipidomics approach using electron capture atmospheric pressure chemical ionization/mass spectrometry [52]. Finally, SFC coupled with Orbitrap mass spectrometry based lipidomics platform was used to identify diverse lipid molecular species [53].

\section{$\underline{\text { Reversed-phase LC }}$}

RPLC is most widely used and separations are based on lipophilicity of interacting components, e.g. shorter carbon chains and polyunsaturated analogs being more polar elute earlier as compared to longer carbon chains and saturated acyl structures, respectively. Before 2004, long narrow (100-250 mm), normal bore (2-4.6 mm I.D.) columns with higher particle size (3.5-5 $\mu \mathrm{m})$ were used. With the advent of UHPLC columns with $2 \mu \mathrm{m}$ particle size were in use with higher flow rates and are resulting in better resolution. Further, to decrease the diffusional mass transfer path, operating at higher speeds and lower back pressures, "fused core" technology was used with 2.6-2.8 $\mu \mathrm{m}$ particles with a 0.35-0.5 $\mu \mathrm{m}$ porous shell fused to a solid core [54]. C18, a core-shell column, showed superior performance in case of chromatographic peak characteristics (plate number, number of detected lipid features) [55]. Few applications focused on miniaturization of lipidomics analyses, employing capillary (50-650 $\times$ 0.15-0.32 mm I.D.; $1.7-5 \mu \mathrm{m})$ and nanobore $(50-170 \times 0.075 \mathrm{~mm}$ I.D.; 3-5 $\mu \mathrm{m})$ columns; as these offer higher sensitivity 
and smaller sample requirements at lower flow-rates of $0.3-10 \mu \mathrm{L} / \mathrm{min}[56]$.

In lipidomics, C18 or C8-modified sorbents based RPLC columns of short length (50-150 mm; typically, $100 \mathrm{~mm}$ ) microbore (1.0-2.1 mm I.D) with particle size of sub-2 $\mu \mathrm{m}$ or 2.6-2.8 $\mu \mathrm{m}$ (fused-core) are majorly used. Examples are the Acquity UPLC BEH C18, Zorbax Eclipse XDB-C18, Acquity UPLC HSS T3, Acquity UPLC BEH C8, Kinetex $\mathrm{C} 18$ and Ascentis Express $\mathrm{C} 8$ columns. These columns can be used at a flowrate of 0.1-0.5 $\mathrm{mL} / \mathrm{min}$ and $40-55^{\circ} \mathrm{C}$ temperatures. Apart from these, $\mathrm{C} 30$ stationary phase are also used, though their use is limited in untargeted profiling of lipidome, but its potential has been demonstrated in separation of phospholipids [57]. The geometric and positional isomers of structurally related lipids having conjugated double bonds can be separated by polymeric C30 stationary phase; this is attributed to phase thickness [58]. A comprehensive untargeted lipidomic analysis using core-shell C30 particle column has been demonstrated by Narváez-Rivas and Zhang [59]. These columns showed an excellent resolution of triglyceride regioisomers, which only differed in fatty acid positions (sn-1, 2 or 3) on the glycerol [60]. Few examples of $\mathrm{C} 30$ columns are Acclaim ${ }^{\mathrm{TM}} \mathrm{C} 30$, Accucore ${ }^{\mathrm{TM}} \mathrm{C} 30, \mathrm{HALO}$ C30. Along these column chemistries, achieved good LC separation and detection of lipids by RPLC; a mixture of water with or without different combinations of mobile phase additives of ammonium formate or acetate (5-10 mM), formic or acetic acid (0.05-0.1\%) and organic solvent(s) like acetonitrile, methanol, isopropanol (IPA) or tetrahydrofuran (THF) have to be used. RPLC has few limitations in separating phospholipids.

\section{Normal-phase LC}

Separation of phospholipids with RPLC is not very efficient, so NPLC is used even though analysis time is more because of long length (100-250 mm; typically, $150 \mathrm{~mm}$ ) though having microbore (2 mm I.D) with 3$5 \mu \mathrm{m}$ particle size and separates lipids based on their polar functional group [56]. Few examples are Luna 3 $\mu \mathrm{m}$ silica, LiChrospher Si 60, Betasil silica-100, and Nucleosil 100-5 OH. These columns are operated at a flow-rate of $0.1-0.5 \mathrm{~mL} / \mathrm{min}$ but with higher flow-rate $(1.0 \mathrm{~mL} / \mathrm{min})$ split mode should be used and maintained at temperatures of $20-35^{\circ} \mathrm{C}$. Mobile phase employed over here are highly non-polar solvents with low ionization capacity like heptane, propan-1-ol, methyl ter-butyl ether, chloroform, ethanol and methanol. Different proportions of these solvents can be used to get weak to strong mobile phases. At times, additives like $0.5 \% \mathrm{NH}_{4} \mathrm{OH} ; 5-15 \mathrm{mM}$ ammonium acetate, ammonium formate, diethylamine, formic acid, or small amounts of water (0.5-3\%) are added to get adequate separation of lipidome.

\section{Hydrophilic interaction chromatography (HILIC)}

HILIC technique offers the benefits of both normal-phase and reverse-phase in lipid separations. HILIC columns exhibited extraordinary separation of lipid classes based on head group composition because of its hydrophilic properties; while using the same RPLC mobile phases to improve ionization efficiency and reproducibility of fatty acid chain length, degree of saturation and double bond position [61].

Lipid separations under HILIC conditions are usually conducted on shorter length (100-150 mm) microbore (2 mm I.D.) columns with 1.7-5 $\mu \mathrm{m}$ particle size, such as Atlantis HILIC silica, Acquity UPLC BEH HILIC, Nucleosil 100-5 OH and Spherisorb Si. These columns are operated at a flow-rate of $0.1-1 \mathrm{~mL} / \mathrm{min}$ and maintained at temperatures of $25-40{ }^{\circ} \mathrm{C}$. The analysis time is typically in the range of $15-60 \mathrm{~min}$. Weak and strong mobile phases are used with high proportion of acetonitrile and water respectively, with methanol and IPA. Additives used are 0.1-0.2 \% formic acid, 5-10 mM ammonium acetate, $20 \mathrm{mM}$ ammonium formate, or $10 \mathrm{mM} \mathrm{NH}_{4} \mathrm{OH}$. It has been demonstrated that HILIC can be used as an alternate system in a mix-mode with reverse phase liquid chromatography in the analysis of complex lipids $[56,57]$.

Extreme diversity and challenges are faced in the analysis of complex lipids (isobars, regioisomers, 
ether-linkage or head group modification). To overcome this, many scientists have combined two liquid chromatography platforms to reduce the sample complexity, analysis times and elevated linear dynamic range [61]. HILIC and C30 reversed-phase chromatography (C30RP) coupled to high resolution mass spectrometry was used to analyse modified class of acylphosphatidylglycerol (acyl PG) in corn roots by HILIC, and further resolution of the isomers was enhanced using C30 RP chromatography [62]. Rampler et al. (2018) had used orthogonal HILIC and RP separations in parallel and the effluents of both columns were combined prior to high-resolution MS detection, to achieve full separation in one analytical run [63].

\section{Long micropillar array columns ( $\mu \mathrm{PAC})$}

This unique technology was developed by a lithographic etching process to create a perfectly ordered separation bed on a silicon chip. Freestanding nature of the pillar offers several advantages compared to conventional columns by elimination of heterogeneous flow paths in the separation bed, thereby low backpressure, high resolution and high sensitivity. Sandra et al. (2017) has demonstrated inter and intra class separation and resolving isomeric lipids [64]. The blood plasma lipid differentiation of lysoglycerophospholids (Lyso - GPs) and monoglycerides (MGs) from the glycerophospholipids (GPs), sphingolipids (SPs) and diglycerides (DGs) was studied [64]. This is commercially available with PharmaFluidics (https://www.pharmafluidics.com).

\section{MALDI and MALDI-ToF mass spectrometry}

MALDI-ToF is an ideal complementary tool for shotgun lipidomic experiments since late 1990, because of its excellent sensitivity, high tolerance against salts, sample impurities, instrument robustness and freedom from crossover sample contamination [65]. Imaging lipids, mapping the distribution of various lipids in different tissues have been successfully carried out by many researchers using this technique which will be discussed later in this review. However, right choice of matrix plays a pivotal role in MALDIToF, for example free fatty acids analysis is difficult with standard matrixes, 2,5-dihydroxy benzoic acid and alfa-cyano-4-hydroxy cinnamic acid, due to the signal and matrix overlap. Instead basic matrixes like 9amino-acridine and 2-mercaptobenzothiazole have been in use. Schiller et al. (1999) had comprehended an article on various conditions and matrices used in this technique [66]. So, MALDI-ToF provides a fast, easy, and useful tool for profiling complex lipid mixture, microbial lipids such as lipid A [67] and phosphatidylinositol mannosides [68]. MALDI-MS can be very easily coupled with thin-layer chromatography (TLC) allowing the spatially resolved screening of the entire TLC plate and the detection of lipids with a higher sensitivity and nondestructively in comparison with IR lasers and UV lasers [69]. Major setback of this method is in the quantitative analysis, due to lack of reproducibility, lack of universal matrix and interference of chemical background noise especially in low mass regions. A combination of highenergy collision-induced dissociation (CID) and prompt ion detection characteristic for MALDI ToF/ToF MS/MS has a unique feature of remote fragmentation of lipids at the level of fatty acyl sn position and double bond location, making the structural analysis easy [70]. Pittenauer et al. (2011) have illustrated many applications using this [71].

\section{Ion Mobility Spectrometry}

Separation of isomeric and isobaric species in complex biological samples is a major challenge in HRMS; either shotgun MS or coupled with liquid chromatography MS. Ion mobility separates ions based on their differential mobility (size, shape, charge) through an inert gas (typically helium, argon or nitrogen) under the influence of an electric field [72,73]. The IM-MS is a strong synergy between these two techniques because of their ability to ascertain complementary information about gas-phase ions. Three-dimensional 
separations are achieved by a range of front-end techniques, IMS and MS providing fast measurements, providing new insights into lipid biology [74]. The front-end analytical separations include gas chromatography, supercritical fluid chromatography, liquid chromatography, solid-phase extractions, capillary electrophoresis, field asymmetric ion mobility spectrometry and microfluidic devices [74]. Whereas MS includes time-of-flight mass spectrometers (ToF-MS), quadrupole mass spectrometers (qMS), ion trap mass spectrometers (IT-MS), Fourier transform mass spectrometers (FTMS) and magnetic sector mass spectrometer. The major ionization sources are electrospray, MALDI and Laser spray ionization. Paglia et al. $(2014,2015)$ have done extensive work in this field and provide incredibly detailed protocols on various IMS-coupled mass spectrometry methods $[75,76]$.

Ion Mobility Spectroscopy technologies

There are four commercially available IMS-MS technologies that have been utilized for lipidomics analysis: (i) Drift Time Ion Mobility Spectrometry (DTIMS) - here packets of ions are injected into a drift tube filled with an inert buffer gas. Under the influence of a weak electric field, ions are separated by charge, size, and shape, developed by Agilent Technologies [77] (ii) Field Asymmetric lon Mobility Spectrometry/Differential Mobility Spectrometry (FAIMS/DMS) - an asymmetric waveform is applied to two cylindrical plates such that ions experience alternating high and low electric fields. Ions traverse the region between the plates moving in a perpendicular direction to the buffer gas and with the influence of a DC potential, termed the compensation voltage (CV). Only selected ions at a given $\mathrm{CV}$ will make it through the drift region, this approach is provided by Sciex [78] (iii) Travelling Wave Ion Mobility Spectrometry (TWIMS) - an alternating phase radio-frequency (RF) potential is applied to a series of stacked ring ion guides (SRIGs). lons are pushed through the drift region with a traveling potential wave and become mobility separated as higher mobility ions are able to 'roll-over' the traveling waves generated and exit the SRIG region, developed by Waters [79] and (iv) Trapped Ion Mobility Spectrometry (TIMS), this uses an electric field to hold ions stationary against a moving gas, so that the drift force is compensated by the electric field and ion packages are separated based on their respective ion mobilities and process called parallel accumulation serial fragmentation or PASEF, developed by Bruker [80]. A detailed description of the theoretical concepts we refer the readers to reviews in this field $[73,81]$.

Improved lipid identification by IM-MS

Identification of lipids has been done by accurate mass match with online databases such as LIPID MAPS or LipidBlast, but it provides only molecular formula. This is inconclusive as number of species belonging to different lipid classes has same molecular formula. The physicochemical characteristics of the compounds are required to allow a more accurate identification. This allows the calculation of the collision cross section (CCS), a four-dimensional orthogonal (retention time, $\mathrm{m} / \mathrm{z}$, ion mobility, intensity) physicochemical measure that can be used, together with accurate mass, fragmentation information and retention time (Rt) to increase the confidence of lipid identification in milliseconds. It is a known fact that, saturated lipids bearing acyl chains are extended in the electric field and have larger CCS values as compared to the unsaturated bonds with bent structure in the acyl chain. The CCS values are more influenced by the structural characteristics of compounds than the degree of saturation [75,82-84]. This was further correlated with the CCS values of FAs and PCs with both the lipid chain length and the degree of unsaturation. Many scientists have determined the CCS values to cover the lipidome of complex biological matrices [83,84]. Catherine et al. compiled 1856 lipid CCS values from plasma, liver and cancer cells with high quality of ${ }^{\text {TIMS }}$ CCS values [85]. Zhou et al. (2017) developed a support vector regression model using bioinformatic approaches and set of molecular descriptors, earnestly describing the subtle structural 
differences for lipids on SMILES structures [86]. These in silico LipidCCS values are independent data and are externally validated from the experimentally (TWIMS and DTIMS) determined values. Lipid CCS Predictor offers (i) prediction of lipid CCS values; (ii) LipidCCS database search and (iii) lipid match and identification [84]. The Lipid CCS database approximately contains over 15,000 lipids with over 60,000 corresponding CCS values determined experimentally or predicted in silico. CCS evaluation an additional identifying factor to improve data interpretation and enhance lipid identification in untargeted workflows [75]. However, validation of CCS values is restricted to the limited number of commercially available lipid standards and can only be used as an in silico CCS prediction to improve identification efficiency in lipidomics.

\section{Applications/Isomer separation}

Ion mobility system is exceptionally well-suited for untargeted lipidomics due to high-resolution, highthroughput and structural elucidation capabilities. Notable separation occurs in ion mobility (IM) before fragmentation (MS), as such product ions are mobility-aligned to corresponding precursor ions thereby improving interpretation of product ion spectra. Also, identical product ions derived from different precursor ions are proportionately assigned to their precursors, enhancing the low-abundance species detection [87]. One of the major confrontation in lipid analysis is isomer separation [88], with regio- [such as sn1 (16:0) or sn2 (18:1 (9Z)) for GPs] [89], positional (position of double bond), or geometric (cis/trans conformation of the double bond i.e. Z/E) isomers [90]. Typical example of glycerophospholipids is shown in Figure 2. Zandkarimi et al. (2019) separated, co-eluted plasmalogen phosphatidylethanolamine (PE p-) PE ( $p-36: 1)$ and PE ( $p-38: 2)$ lipids in mice brain tissue using LC-IM-MS. As both the PE had same retention time but were separated clearly in the ion mobility region with different drift time bin number [91]. Thus, structural elucidation was feasible due to IMS drift time, high collisional energy in transfer region and clear fragmentation pattern [76].

Technical developments in both hardware and software, empowers researchers to implement IM-MS into their analytical workflows. The four major augmentations in lipidomics are firstly, IM-MS crucially resolves isobaric species, thereby improves separation of lipids. Secondly, IM fragmentation improves the spectral clarity of product ion spectra. This is crucial in both lipid identification and structural elucidation, which are grueling task due to the isomeric nature of many lipid species. On the other hand, analysis of product ion spectra derived from untargeted fragmentation acquisitions remains challenging due to the required powerful processing tools. Thirdly, IM improves separation of isomeric lipids. Lastly, CCS values obtained from IM-MS analysis effectively increase confidence in lipid identification. Finally, IM-MS can be used to comprehend the conformational dynamics of a lipid system and offering a unique means of characterizing flexibility and folding mechanisms.

\section{MS Lipidomics Imaging and in situ}

Lipidomics provide spatial information about the lipid composition in tissues - sort of molecular microscope [92]. There are several desorption ionizations tools and imaging MS techniques but 'Lipidomics standard initiative' (https://lipidomics-standards-initiative.org/) have recommended only secondary ion mass spectrometry (SIMS), desorption electrospray ionization (DESI) and matrix-assisted laser desorption/ionization (MALDI). Out of which, MALDI-imaging mass spectrometry (MALDI-IMS) is commonly used for lipid imaging in tissue sections. 

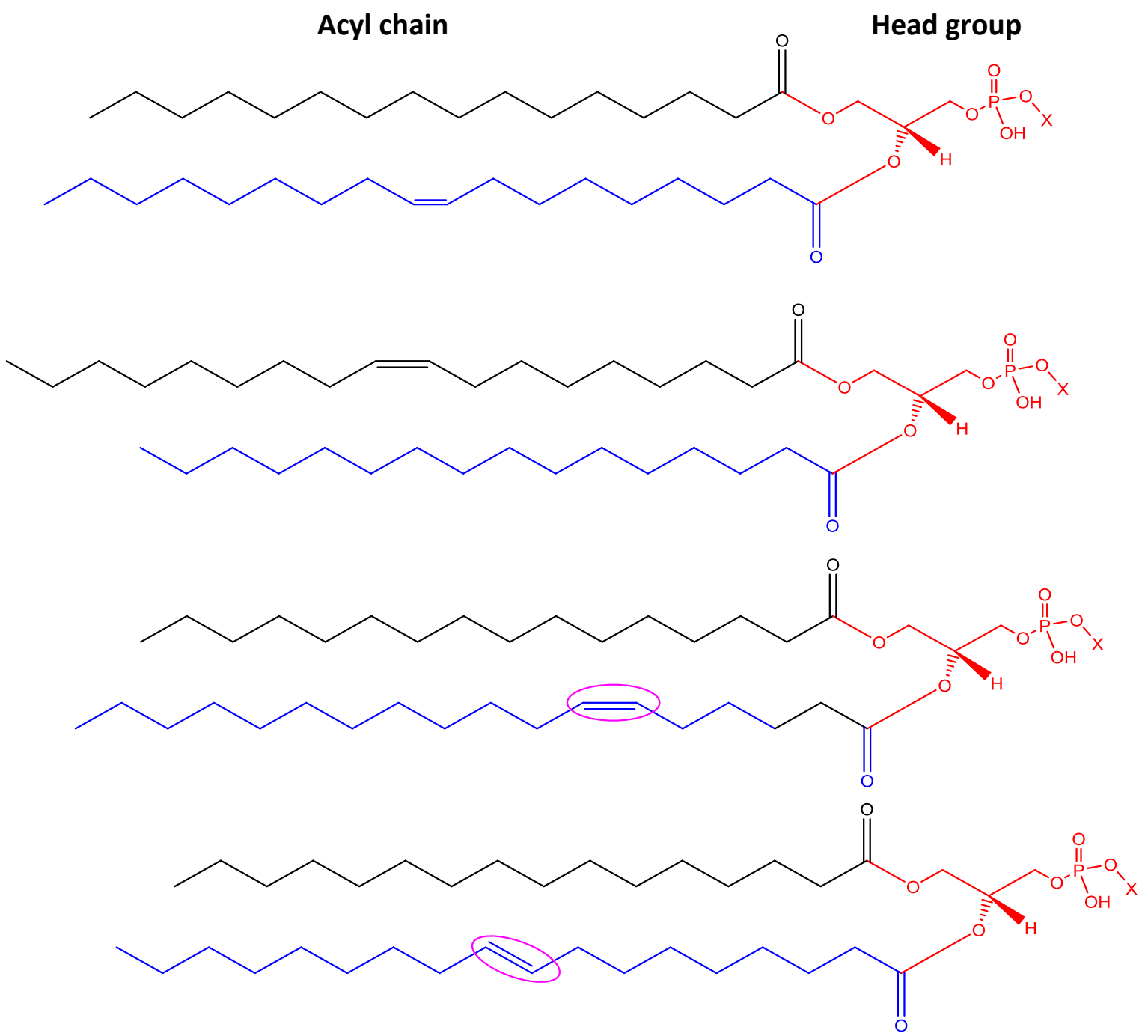

Figure 2. Isomeric structures of glycerophospholipids [Polar head group indicated as ' $x$ ' can be glycerol, choline, ethanolamine, inositol, or serine and two fatty acyl groups at sn1 (16:0) and sn2 (18:1 (9Z)) position forming the GP tail. Three types of isomers are represented - regioisomer presenting the fatty acyl chains in inverted positions; a positional isomer, containing the double bond in a different position (C6 instead of C9), and a geometric isomer, whose double bond is in trans (E) conformation]

\section{MALDI-imaging mass spectrometry (MALDI-IMS)}

MALDI-imaging mass spectrometry (MALDI-IMS) is a two-dimensional MALDI-MS technique which gives comprehensive profiles of molecular distributions of lipids [93] with high spatial resolution without extraction, purification, separation, or labeling of biological samples. This reveals the localization and abundance of hundreds of molecular species, especially lipids on the tissue slice in a single measurement thus helping in understanding the cellular profile of the biological system. Shimma et al. (2007) used this study the abnormal distribution of phospholipids in colon cancer liver metastasis [93].

This technique is widely used in brain and skin lipidomics as well [94]. Goto-Inoue et al. (2011) have used TLC-Blot-MALDI-IMS to study detailed structural analysis of lipids from human brain samples [95]. This was done by a three-step process, by running TLC once, transferring to a polyvinylidene difluoride membrane and finally detection by MALDI-IMS. Thus it was possible to separate, visualize and identify phosphatidylserine (PS) (diacyl-18:0/20:4), phosphatidylcholine (PC) (diacyl-16:0/18:1), and sphingomyelin (SM) (d18:1/C18:0) at $m / z$ 812.5, 753.5 and 782.5 in gray matter. It was proposed that, this system would be useful in fully analyzing lipid compositions including minor components. Kendall et al. applied this 
technique in skin lipidomics [95] by analyzing ex vivo human skin cutaneous lipids for assessing alterations in lipid profiles linked to specific skin conditions. Both MALDI and DESI skin imaging are used for analysis of the whole skin sections, though the analysis time was same, disadvantages being, the former had matrix selection and later had less spatial resolution. SIMS imaging is known for high spatial resolution but a low mass range, which can decipher the spatial distribution of multiple lipids at subcellular levels. A completely new technique was studied using Infrared matrix assisted laser desorption electrospray ionization mass spectrometry (IR-MALDESI), combines many benefits of MALDI and ESI. IR-MALDESI works by combining laser desorption of neutrals with subsequent ionization by ESI to increase lipid abundance with great coverage relative to commonly used parameters in negative polarity [96]. This technique can be used for quantitative analysis and ideally for drug distribution studies [96].

The use of other ambient ionization tools, including rapid evaporative ionization mass spectrometry (REIMS) and direct analysis in real time (DART) allow rapid, real-time screenings of lipids for predictive, preventive, and personalized medicine. REIMS based methods require no preparative steps or timeconsuming cell extractions which are discussed later in this review. IR-MALDESI sensitivity and selectivity was augmented using silver cationization of olefinic lipids in human serum using calcifediol as the reference [97].

\section{Rapid Evaporative ionization mass spectrometry (REIMS)}

REIMS is truly ambient analysis technique, for fast, easy molecular profiling with zero sample preparation. This is highly versatile technique as it can be used for both biological solid and liquid samples, as this provides quick determination of differences within and between samples. This is achieved by simple evaporation of the sample by Joule heating or laser irradiation, the aerosol generated is introduced orthogonally to the inlet of the mass spectrometer such as high performance ToF-MS. This technique has been used in intra-surgical tissue classification [98], bacterial identification [96], rapid profiling of cell lines [100] the analysis of plant material, food applications [101] and bioliquid samples. This technique has a potential to combine with other sampling techniques in providing a holistic profiling approach.

Few scientists have used matrix assisted version of the technique (MA-REIMS) to enhance the signal intensity in identifying strong phospholipid signal in the tumor which is absent in normal breast tissue [98]. In another group, in situ and real-time recognition model was employed in identifying 12 fatty acids and 37 phospholipids using "iKnife" and REIMS in discrimination of salmon and rainbow trout without sample preparation and adulteration of minced meats [102]. Despite so many advantages, the major setback of this technique is, it provides only moderate amounts of chemical information and repeatability, for further information we should still bank on LC-MS.

\section{Direct analysis in real time-mass spectrometry (DART-MS)}

DART-MS is an ambient pressure ionization technique enabling instantaneous and sensitive analyses of gases, liquids and solids [103]. It is based on the interactions of long lived electronic or vibronic excitedstate molecule with sample and atmospheric gases at atmospheric pressure. This technique does not require laborious sample preparation, as ionization takes place directly on the sample surface, deposited or adsorbed on to surfaces or that are being desorbed into the atmosphere. The combination of this source with a high-resolution mass spectrometer (commercially available with JOEL as DART-Accu TOF) offers a rapid qualitative and quantitative measurements. It has numerous applications in the field of food science, forensics, and clinical analysis (https://www.jeol.co.jp/en/applications/pdf/ms/ms_note_en002.pdf). “Noprep" analysis of lipids in cooking oils and detection of adulterated olive oil is an application using DART- 
Accu TOF. Despite numerous advantages, DART ionization does have several inherent limitations. Firstly, fragmentation occurs at higher plasma temperatures, hindering spectra interpretation and accurate determination of the mass of intact molecules, well the decomposition fragments can also contribute to the structural information [104]. Secondly, this technique subjects the analyte to oxidation artifacts, owing to the design of the instrument i.e., distance from the capillary outlet [104]. Lastly, saturated hydrocarbons can undergo hydride abstraction hindering the quantitative analysis. For example, signals from aliphatic hydrocarbons and monounsaturated hydrocarbons are indistinguishable due to the same carbon length [105].

\section{Conclusions}

In this review, we have outlined different analytical strategies for a comprehensive identification, complete structural characterization and accurate quantitation of biogenic lipid molecules with a fewer bottle necks. It must be noted, that each technique has its own strengths and weaknesses for example high mass resolution results in accurate mass but with natural limitations. Therefore, a mixture of analytical devices (chromatography, spectrometry, mass spectrometry and hyphenated methods) helps to cope up with the complexity of the lipids structure. However, lipidomics provide enormous data especially nontargeted lipids and it is critical to evaluate it using bioinformatics solutions [106]. The lipid information is available as web resources namely, cyberlipid center (http://www.cyberlipid.org/) and AOCS lipid library (http://lipidlibrary.aocs. org/). Tools that have been developed for analysis of MS-based lipidomic data include MS and MS/MS data by lipid consortium such as LIPID MAPS Lipidomics Gateway (www.lipidmaps.org/resources/tutorials/databases.html) and National Institute of Standards and Technology (NIST) (http://chemdata.nist.gov/); and commercialized software such as Lipidview ${ }^{\text {TM }}$ (https://sciex.com/products/software/lipidview-software), LipidSearch ${ }^{\text {TM }}$ (https://www.thermofisher.com/ hr/en/home/industrial/mass-spectrometry/liquid-chromatography-mass-spectrometry-lc-ms/lc-ms-

software.html), and SimLipid (www.premierbiosoft.com) also become available. This will undoubtedly be a valuable tool for investigation of many diseases, physiological processes or in disease biomarker discovery.

Conflict of interest: Ramesh Mullangi is Vice President, Pre-clinical Biology \& DMP at Laxai Life Sciences.

\section{References}

[1] E. Fahy, S. Subramaniam, R.C. Murphy, M. Nishijima, C.R.H. Raetz, T. Shimizu, F. Spener, G. Meer, M.J.O. Wakelam, E.A. Dennis. Update of the LIPID MAPS comprehensive classification system for lipids. Journal of Lipid Research 50 (2009) S9-S14. doi: https://doi.org/10.1194/ilr.R800095JLR200.

[2] E. Fahy, D. Cotter, M. Sud, S. Subramaniam. Lipid classification, structures and tools. Biochim. Biophys. Acta 1811 (2001) 637-647. https://doi.org/10.1016/j.bbalip.2011.06.009.

[3] X. Wang, D. Wu, H. Shen. Lipidomics in health and disease: methods and application. In: X.D. Wang (ed). Translational Bioinformatics, vol 16. Berlin: Springer, 2018.

[4] G.B. Mills, W.H. Moolenaar. The emerging role of lysophosphatidic acid in cancer. Nature Reviews Cancer 3 (2003) 582-591. https://doi.org/10.1038/nrc1143.

[5] R.G. Cutler, J. Kelly, K. Storie, W.A. Pedersen, A. Tammara, K. Hatanppa, J.C. Troncoso, M.P. Mattson. Involvement of oxidative stress-induced abnormalities in ceramide and cholesterol metabolism in brain aging and Alzheimer's disease. Proceedings of the National Academy of Sciences USA 101 (2004) 2070-2075. https://doi.org/10.1073/pnas.0305799101.

[6] T. Murakami, T. Urata, N. Yamada, K. Kawakubo, T. Yoshida, H. Kawamura, T. Kashiwa, S. Lino. Lipoprotein abnormalities in type 2 diabetic patients. Increased distribution of triglycerides (TG) in 
very low density lipoprotein (VLDL) and decreased apolipoprotein E/VLDL-TG ratio are risk for ischemic heart disease. Artery 22 (1997) 309-327.

[7] J.Z. Kubicek-Sutherland, D.M. Vu, H.M. Mendez, S. Jakhar, H. Mukundan. Detection of lipid and amphiphilic biomarkers for disease diagnostics. Biosensors 7 (2017) 25. https://doi.org/10.3390/ bios7030025.

[8] E.C.P. Chua, G. Shui, I.T. Lee, P. Lau, L.C. Tan, S.C. Yeo, B.D. Lam, S. Bulchand, S.C. Summers, K. Puvanendran, S.G. Rozen, M.R. Wenk, J.J. Gooley. Extensive diversity in circadian regulation of plasma lipids and evidence for different circadian metabolic phenotypes in humans. Proceedings of the National Academy of Sciences USA 110 (2013) 14468-14473. https://doi.org/10.1073/ pnas.1222647110.

[9] M. Scherer, G. Schmitz, G. Liebisch. High-throughput analysis of sphingosine 1-phosphate, sphinganine 1-phosphate and lysophosphatidic acid in plasma samples by liquid chromatographytandem mass spectrometry. Clinical Chemistry 55 (2009) 1218-1222. https://doi.org/10.1373/clin chem.2008.113779.

[10] J. Kim, C.L. Hoppel. Comprehensive approach to the quantitative analysis of mitochondrial phospholipids by HPLC-MS. Journal of Chromatography B 912 (2013) 105-114. https://doi.org/10. 1016/j.jchromb.2012.10.036.

[11] M. Okudaira, A. Inoue, A. Shuto, K. Nakanaga, K. Kano, K. Makide, D. Saigusa, Y. Tomioka, J. Aoki. Separation and quantification of 2-acyl-1-lysophospholipids and 1-acyl-2-lysophospholipids in biological samples by LC-MS/MS. Journal of Lipid Research 55 (2014) 2178-2192. doi: https://doi.org L10.1194/jlr.D048439.

[12] U. Ceglarek, J. Dittrich, C. Helmschrodt, K. Wagner, J.R. Nofer, J. Thiery, S. Becker. Preanalytical standardization of sphingosine-1-phosphate, sphinganine-1-phosphate and sphingosine analysis in human plasma by liquid chromatography-tandem mass spectrometry. Clinica Chim Acta 435 (2014) 1-6. https://doi.org/10.1016/i.cca.2014.04.010.

[13] J. Dorow, S. Becker, L. Kortz, J. Thiery, S. Hauschildt, U. Ceglarek. Preanalytical investigation of polyunsaturated fatty acids and ecosanoids in human plasma by liquid chromatography-tandem mass spectrometry. Biopreservation and Biobanking 14 (2016) 107-113. https://doi.org/10.1089 $\angle$ bio.2015.0005.

[14] L.A. Heiskanen, M. Suoniemi, H.X. Ta, K. Tarasov, K. Ekroos. Long-term performance and stability of molecular shotgun lipidomic analysis of human plasma samples. Analytical Chemistry 85 (2013) 8757 - 8763. https://doi.org/10.1021/ac401857a.

[15] K. Jurowski, K. Kochan, J. Walczak, M. Barańska, W. Piekoszewski, B. Buszewski. Analytical techniques in lipidomics: State of the art. Critical Reviews in Analytical Chemistry 47 (2017) 418-437. https://doi.org/10.1080/10408347.2017.1310613.

[16] C.Z. Ulmer, R.E. Patterson, J.P. Koelmel, T.J. Garrett, R.A. Yost. A robust lipidomics workflow for mammalian cells, plasma, and tissue using liquid-chromatography high-resolution tandem mass spectrometry. Methods in Molecular Biology 1609 (2017) 91-106. doi: https:/doi.org/10.1007/978-14939-6996-8 10.

[17] J. Folch, M. Lees, G.H. Sloane Stanly. A simple method for the isolation and purification of total lipids from animal tissues. Journal of Biological Chemistry 226 (1957) 497-509.

[18] J. Bligh, M. Lees, G.H. Sloane Stanley. A rapid method of total lipid extraction and purification. Canadian Journal of Biochemistry and Physiology 37 (1959) 911-917.

[19] V. Matyash, G. Liebisch, T.V. Kurzchalia, A. Shevchenko, D. Schwudke. Lipid extraction by methyl-tertbutyl ether for high-throughput lipidomics. Journal of Lipid Research 49 (2008) 1137-1146. Doi: https://doi.org/10.1194/ilr.D700041-JLR200.

[20] R.M. Pellegrino, A. Di Veroli, A. Valeri, L. Goracci, G. Cruciani. LC/MS lipid profiling from human serum: a new method for global lipid extraction. Analytical and Bioanalytical Chemistry 406 (2014) 7937-7948. https://doi.org/10.1007/s00216-014-8255-0. 
[21] D.L. Baker, D.M. Desiderio, D.D. Miller, B. Tolley, G.J. Tigyi. Direct Quantitative analysis of lysophosphatidic acid molecular species by stable isotope dilution electrospray liquid chromatography-mass spectrometry. Analytical Biochemistry 292 (2001) 287-295. https://doi.org/ 10.1006/abio.2001.5063.

[22] W.J. Griffiths, I. Gilmore, E. Yutuc, J. Abdel-Khalik, P.J. Crick, T. Hearn, A. Dickson, B.W. Bigger, T.H.Y. $\mathrm{Wu}, \mathrm{A}$. Goenka, A. Ghosh, S.A. Jones, Y. Wang. Identification of unusual oxysterols and bile acids with 7-oxo or $3 \beta, 5 \alpha, 6 \beta$-trihydroxy functions in human plasma by charge-tagging mass spectrometry with multistage fragmentation. Journal of Lipid Research 59 (2018) 1058-1070. doi: https://doi.org/ 10.1194/jilr.D083246.

[23] M. Wang, J.P. Palavicini, A. Cseresznye, X. Han. Strategy for quantitative analysis of isomeric bis(monoacylglycero)phosphate and phosphatidylglycerol species by shotgun lipidomics after onestep methylation. Analytical Chemistry 89 (2017) 8490-8495. https://doi.org/10.1021/acs.analchem. 7b02058.

[24] J. Clark, K.E. Anderson, V. Juvin, T.S. Smith, F. Karpe, M.J.O. Wakelam, L.R. Stephens, P.T. Hawkins. Quantification of PtdlnsP3 molecular species in cells and tissues by mass spectrometry. Nature Methods 8 (2011) 267-272. Doi: https://doi.org/10.1038/nmeth.1564.

[25] J.C. Lee, S.K. Byeon, M.H. Moon. Relative Quantification of phospholipids based on isotope-labeled methylation by nano flow ultra high performance liquid chromatography-tandem mass spectrometry: Enhancement in cardiolipin profiling. Analytical Chemistry 89 (2017) 49694977. https://doi.org/10.1021/acs.analchem.7b00297.

[26] C. Hu, M. Wang, Q. Duan, X. Han. Sensitive analysis of fatty acid esters of hydroxy fatty acids in biological lipid extracts by shotgun lipidomics after one-step derivatization Analytica Chimica Acta 1105 (2020) 105-111. https://doi.org/10.1016/j.aca.2020.01.026.

[27] X. Zhao, S. Zhu, H. Liu. Recent progresses of derivatization approaches in the targeted lipidomics analysis by mass spectrometry. Journal of Separation Science 43 (2020) 1838-1846. https://doi.org 110.1002/jssc.201901346.

[28] R. Deems, M.W. Buczynski, R. Bowers-Gentry, R. Harkewicz, E.A. Dennis. Detection and quantitation of eicosanoids via high performance liquid chromatography-electrospray ionization-mass spectrometry. Methods in Enzymology 432 (2007) 59-82. Doi: 10.1016/s0076-6879(07)32003-x.

[29] X. Han, K. Yang, R.W. Gross. Multi-dimensional mass spectrometry-based shotgun lipidomics and novel strategies for lipidomic analyses. Mass Spectrometry Reviews 31 (2011) 134-178. https://doi .org/10.1002/mas.20342.

[30] M. Wang, C. Wang, X. Han. Selection of internal standards for accurate quantification of complex lipid species in biological extracts by electrospray ionization mass spectrometry - What, how and why? Mass Spectrometry Reviews 36 (2017) 693-714. Doi: http://doi.org/10.1002/mas.21492.

[31] T. Züllig, M. Trötzmüller, H.C. Köfeler. Lipidomics from sample preparation to data analysis: a primer. Analytical Bioanalytical Chemistry 412 (2020) 2191-2209. https://doi.org/10.1007/s00216019-02241-y.

[32] S. Sales, J. Graessler, S. Ciucci, R. Al-Atrib, T. Vihervaara, K. Schuhmann, D. Kauhanen, M. Sysi-Aho, S.R. Bornstein, M. Bickle, C.V. Cannistraci, K. Ekroos, A. Shevchenko. Gender, contraceptives and individual metabolic predisposition shape a healthy plasma lipidome. Scientific Reports 6 (2016) 27710. https://doi.org/10.1038/srep27710.

[33] X. Han, R.W. Gross. Shotgun lipidomics: Electrospray ionization mass spectrometric analysis and quantitation of cellular lipidomes directly from crude extracts of biological samples. Mass Spectrometry Reviews 24 (2005) 367-412.

[34] X. Han. Characterization and Direct Quantitation of ceramide molecular species from lipid extracts of biological samples by electrospray ionization tandem mass spectrometry. Analytical Biochemistry 302 (2002) 199-212. https://doi.org/10.1006/abio.2001.5536. 
[35] X. Han, R.W. Gross. Electrospray ionization mass spectroscopic analysis of human erythrocyte plasma membrane phospholipids. Proceedings of the National Academy of Sciences USA 91 (1994) 1063510639. Doi: https://doi.org/10.1073/pnas.91.22.10635.

[36] K. Yang, Z. Zhao, R.W. Gross, X. Han. Systematic analysis of choline-containing phospholipids using multi-dimensional mass spectrometry-based shotgun lipidomics. Journal of Chromatography B 877 (2009) 2924-2936. https://doi.org/10.1016/j.jchromb.2009.01.016.

[37] X. Han, R.W. Gross. Shotgun lipidomics: multidimensional MS analysis of cellular lipidomes. Expert Review of Proteomics 2 (2005) 253-264. https://doi.org/10.1586/14789450.2.2.253.

[38] M. Koivusalo, P. Haimi, L. Heikinheimo, R. Kostiainen, P. Somerharju. Quantitative determination of phospholipid compositions by ESI-MS: effects of acyl chain length, unsaturation, and lipid concentration on instrument response. Journal of Lipid Research 42 (2001) 663-672.

[39] X.L. Guan, X. He, W.Y. Ong, W.K. Yeo, G.H. Shui, M.R. Wenk. Non-targeted profiling of lipids during kainate-induced neuronal injury. FASEB Journal 20 (2006) 1152-1161. https://doi.org/10.1096/fj.055362com.

[40] P.R.S. Baker, A.M. Armando, J.L. Campbell, O. Quehenberger, E.A. Dennis. Three-dimensional enhanced lipidomics analysis combining UPLC, differential ion mobility spectrometry and mass spectrometric separation strategies. Journal of Lipid Research 55 (2004) 2432-2442. https://doi.org/ 10.1194/ilr.D051581.

[41] P. Wiesner, K. Leidl, A. Boettcher, G. Schmitz, G. Liebisch. Lipid profiling of FPLC-separated lipoprotein fractions by electrospray ionization tandem mass spectrometry. Journal of Lipid Research 50 (2008) 574-585. Doi: https://doi.org/10.1194/ilr.D800028-JLR200.

[42] G. Liebisch, B. Lieser, J. Rathenberg, W. Drobnik, G. Schmitz. High-throughput quantification of phosphatidylcholine and sphingomyelin by electrospray ionization tandem mass spectrometry coupled with isotope correction algorithm. Biochimica Biophysica Acta 1686 (2004) 108-117. Doi: https://doi.org/10.1016/j.bbalip.2004.09.003.

[43] G. Liebisch, J.A. Vizcaíno, H. Köfeler, M. Trotzmuller, W.J. Griffiths, G. Schmitz, F. Spener, M.J.O. Wakelam. Shorthand notation for lipid structures derived from mass spectrometry. Journal of Lipid Research 50 (2013)1523-1530. Doi: https://doi.org/10.1194/jlr.M033506.

[44] R. Almeida, J.K. Pauling, E. Sokol, H.K. Hannibal-Bach, C.S. Ejsing. Comprehensive lipidome analysis by shotgun lipidomics on a hybrid quadrupole-orbitrap-linear ion trap mass spectrometer. Journal of The American Society for Mass Spectrometry 26 (2014) 133-148. https://doi.org/10.1007/s13361014-1013-x.

[45] M. Höring, C.S. Ejsing, M. Hermansson, G. Liebisch. Quantification of cholesterol and cholesteryl ester by direct flow injection high resolution FTMS utilizing species-specific response factors. Analytical Chemistry 91 (2019) 3459-3466. https://doi.org/10.1021/acs.analchem.8b05013.

[46] J.M. Choi, T.E. Kim, J.Y. Cho, H.J. Lee, B.H. Jung. Development of lipidomic platform and phosphatidylcholine retention time index for lipid profiling of rosuvastatin treated human plasma. Journal of Chromatography B 944 (2014) 157-165. Doi: https://doi.org/10.1016/i.jchromb. 2013.10.029.

[47] F. Gao, J. McDaniel, E.Y. Chen, H.E. Rockwell, C. Nguyen, M.D. Lynes, Y.H. Tseng, R. Sarangarajan, N.R. Narain, M.A. Kiebish. Adapted MS/MS ${ }^{\text {ALL }}$ shotgun lipidomics approach for analysis of cardiolipin molecular species. Lipids 53 (2018) 133-142. doi: https://dx.doi.org/10.1002/lipd.12004 .

[48] S.E. Flaherty, A. Grijalva, X. Xu, E. Ables, A. Nomani, A.W. Ferrante. A lipase-independent pathway of lipid release and immune modulation by adipocytes. Science 363 (2019) 989-993. Doi: https://doi.org $\angle 10.1126 /$ science.aaw2586.

[49] M. Holčapek, R. Jirásko, M. Lísa. Recent developments in liquid chromatography-mass spectrometry and related techniques. Journal of Chromatography A 1259 (2012) 3-15. Doi: https://doi.org/10.1016 Li.chroma.2012.08.072. 
[50] J.T. Lin, C.L. Woodruff, T.A. McKeon. Non-aqueous reversed-phase high-performance liquid chromatography of synthetic triacylglycerols and diacylglycerols. Journal of Chromatography A 782 (1997) 41-48. https://doi.org/10.1016/S0021-9673(97)00482-2.

[51] M. Lísa, K. Netušilová, L. Franěk, H. Dvořáková, V. Vrkoslav, M. Holčapek. Characterization of fatty acid and triacylglycerol composition in animal fats using silver-ion and non-aqueous reversed-phase high-performance liquid chromatography/mass spectrometry and gas chromatography/flame ionization detection. Journal of Chromatography A 1218 (2011) 7499-7510. Doi: https://doi.org/10 .1016/j.chroma.2011.07.032.

[52] S.H. Lee, I.A Blair. Targeted chiral lipidomics analysis of bioactive eicosanoid lipids in cellular systems. BMB Reports 42 (2009) 401-410.

[53] T. Yamada, T. Uchikata, S. Sakamoto, Y. Yokoi, S. Nishiumi, M. Yoshida, E. Fukusaki, T. Bamba. Supercritical fluid chromatography/Orbitrap mass spectrometry based lipidomics platform coupled with automated lipid identification software for accurate lipid profiling. Journal of Chromatography $A$ 1301 (2013) 237-242. Doi: https://doi.org/10.1016/i.chroma.2013.05.057.

[54] D. Guillarme, J. Ruta, S. Rudaz, J.L. Veuthey. New trends in fast and high-resolution liquid chromatography: a critical comparison of existing approaches. Analytical and Bioanalytical Chemistry 397 (2009) 1069-1082. https://doi.org/10.1007/s00216-009-3305-8.

[55] J.O. Omamogho, J.D. Glennon. Comparison between the efficiencies of sub-2 $\mu \mathrm{m} \mathrm{C18}$ particles packed in narrow bore columns. Analytical Chemistry 83 (2011) 1547-1556. Doi: https://doi.org/10 .1021/ac102139a.

[56] T. Cajka, O. Fiehn. Comprehensive analysis of lipids in biological systems by liquid chromatographymass spectrometry. Trends Analytical Chemistry 61 (2014) 192-206. Doi: https://doi.org/10.1016 Li.trac.2014.04.017.

[57] H. Houjou, K. Yamatani, M. Imagawa, T. Shimizu, R. Taguchi. A shotgun tandem mass spectrometric analysis of phospholipids with normal-phase and/or reverse-phase liquid chromatography/electrospray ionization mass spectrometry. Rapid Communications in Mass Spectrometry 19 (2005) 654-666. https://doi.org/10.1002/rcm.1836.

[58] R. Kiyonami, D. Peake, D. Drew, X. Sun, X. Liu, K. Miller.Large-scale lipid profiling of a human serum lipidome using a high-resolution, accurate-mass LC-MS/MS approach. Thermo Scientific 2016; Application note 647.

[59] M. Narváez-Rivas, Q. Zhang. Comprehensive untargeted lipidomic analysis using core-shell C30 particle column and high field orbitrap mass spectrometer. Journal of Chromatography A 1440 (2016) 123-134. https://doi.org/10.1016/j.chroma.2016.02.054.

[60] J.W. Lee, T. Nagai, N. Gotoh, E. Fukusaki, T. Bamba. Profiling of regioisomeric triacylglycerols in edible oils by supercritical fluid chromatography/tandem mass spectrometry. Journal of Chromatography B 966 (2014) 193-199. https://doi.org/10.1016/i.jchromb.2014.01.040.

[61] M. Lisa, E. Cifkova, M. Holcapek. Lipidomic profiling of biological tissues using off-line twodimensional high-performance liquid chromatography-mass spectrometry. Journal of Chromatography A 1218 (2011) 5146-5156. https://doi.org/10.1016/i.chroma.2011.05.081.

[62] T.H. Pham, M. Zaeem, T.A. Fillier, M. Nadeem, N.P. Vidal, C. Manful, S. Cheema, M. Cheema, R.H. Thomas. Targeting modified lipids during routine lipidomics analysis using HILIC and C30 reverse phase liquid chromatography coupled to mass spectrometry. Scientific Reports 9 (2019) 5048. https://doi.org/10.1038/s41598-019-41556-9.

[63] E. Rampler, H. Schoeny, B.M. Mitic, Y. El Abiead, M. Schwaiger, G. Koellensperger. Simultaneous nonpolar and polar lipid analysis by on-line combination of HILIC, RP and high resolution MS. Analyst 143 (2018) 1250-1258.

[64] K. Sandra, P. Sandra, W. D. Malsche, G. Desmet, R. t'Kindt R, J.O.D. Beeck, B. Claerebout, J. Vandenbussche. Evaluation of micro-pillar array columns $\left(\mu \mathrm{PAC} C^{\mathrm{TM}}\right)$ combined with high resolution mass spectrometry for lipidomics. LC GC Europe HPLC 30 (2017) 6-13. 
[65] S. Shimma, Y. Sugiura, T. Hayasaka, Y. Hoshikawa, T. Noda, M. Setou. MALDI-based imaging ass spectrometry revealed abnormal distribution of phospholipids in colon cancer liver metastasis. Journal of Chromatography B 855 (2007) 98-103. https://doi.org/10.1016/i.jchromb.2007.02.037.

[66] J. Schiller, J. Arnhold, S. Benard, M. Müller, S. Reichl, K. Arnold. Lipid analysis by matrix-assisted laser desorption and ionization mass spectrometry: A Methodological Approach. Analytical Biochemistry 267 (1999) 46-56. https://doi.org/10.1006/abio.1998.3001.

[67] P. Zhou, E. Altman, M.B. Perry, J. Li. Study of matrix additives for sensitive analysis of Lipid a by matrix-assisted laser desorption ionization mass spectrometry. Applied and Environmental Microbiology 76 (2010) 3437-3443. Doi: https://doi.org/10.1128/AEM.03082-09.

[68] F.F. Hsu, J. Turk, R.M. Owens, E.R. Rhoades, D.G. Russell. Structural characterization of phosphatidylmyo-inositol mannosides from Mycobacterium bovis bacillus calmette gúerin by multiple-stage quadrupole ion-trap mass spectrometry with electrospray ionization. II. Monoacyl- and diacyl-PIMs. Journal of the American Society for Mass Spectrometry 18 (2007) 479-492. https://doi.org/10 .1016/i.jasms.2006.10.020.

[69] K. Dreisewerd, J. Müthing, A. Rohlfin, I. Meisen, Z. Vukelić, J. Peter-Katalinić, F. Hillenkamp, S. Berkenkamp. Analysis of gangliosides directly from thin-layer chromatography plates by infrared matrix-assisted laser desorption/ionization orthogonal time-of-flight mass spectrometry with a glycerol matrix. Analytical Chemistry 77 (2005) 4098-4107. https://doi.org/10.1021/ac048373w.

[70] C. Cheng, E. Pittenauer, M.L. Gross. Charge-remote fragmentations are energy-dependent processes. Journal of The American Society for Mass Spectrometry 9 (1988) 840-844. https://doi.org/10.1016/ S1044-0305(98)00053-1.

[71] E. Pittenauer, G. Allmaier. A universal product ion nomenclature for $[M-H]^{-},[M+H]^{+}$and $[\mathrm{M}+\mathrm{nNa}-(\mathrm{n}-1) \mathrm{H}]+(\mathrm{n}=1-3)$ glycerophospholipid precursor ions based on high-energy CID by MALDITOF/RTOF mass spectrometry. International Journal of Mass Spectrometry 301 (2011) 90101. https://doi.org/10.1016/j.ijms.2010.07.012.

[72] C. Lapthorn, F. Pullen, B.Z. Chowdhry. Ion mobility spectrometry-mass spectrometry (IMS-MS) of small molecules: separating and assigning structures to ions. Mass Spectrometry Reviews 32 (2013) 43-71. DOI: https://doi.org/10.1002/mas.21349.

[73] J.C. May, J.A. McLean. Ion Mobility-Mass Spectrometry: time-dispersive instrumentation. Analytical Chemistry 87 (2015) 1422-1436. https://doi.org/10.1021/ac504720m.

[74] X. Zheng, R. Wojcik, X. Zhang, Y.M. Ibrahim, K.E. Burnum-Johnson, D.J. Orton, M.E. Monroe, R.J. Moore, R.D, Smith, E.S Baker. Coupling front-end separations, ion mobility spectrometry, and mass spectrometry for enhanced multidimensional biological and environmental analyses. Annual Review of Analytical Chemistry 10 (2017) 71-92. https://doi.org/10.1146/annurev-anchem-061516-045212.

[75] G. Paglia, P. Angel, J.P. Williams, K. Richardson, H.J. Olivos, J. Will Thompson, L. Menikarachchi, S. Lai, C. Walsh, A. Moseley, R.S. Plumb, D.F. Grant, B.O. Palsson, J. Langridge, S. Geromanos, G. Astarita. Ion mobility-derived collision cross section as an additional measure for lipid fingerprinting and identification. Analytical Chemistry 87 (2014) 1137-1144. https://doi.org/10.1021/ac500405x.

[76] G. Paglia, M. Kliman, E. Claude, S. Geromanos, G. Astarita. Applications of ion-mobility mass spectrometry for lipid analysis. Analytical and Bioanalytical Chemistry 407 (2015) 4995-5007. Doi: https://doi.org/10.1007/s00216-015-8664-8.

[77] Y.M. Ibrahim, E.S. Baker, W.F. Danielson, R.V. Norheim, D.C. Prior, G.A. Anderson, M.E Belov, R.D. Smith. Development of a new ion mobility time-of-flight mass spectrometer. International Journal of Mass Spectrometry 377 (2015) 655-662. https://doi.org/10.1016/j.ijms.2014.07.034.

[78] B.B. Schneider, E.G. Nazarov, F. Londry, P. Vouros, T.R. Covey. Differential mobility spectrometry/ mass spectrometry history, theory, design optimization, simulations, and applications. Mass Spectrometry Reviews 35 (2016) 687-737. https://doi.org/10.1002/mas.21453.

[79] A.A. Shvartsburg, R.D. Smith. Fundamentals of traveling wave ion mobility spectrometry. Analytical Chemistry 80 (2008) 9689-9699. https://doi.org/10.1021/ac8016295. 
[80] F. Fernandez-Lima, D.A. Kaplan, J. Suetering, M.A Park. Gas-phase separation using a trapped ion mobility spectrometer. International Journal of Ion Mobility Spectrometry 14 (2011) 93-98. https://doi.org/10.1007/s12127-011-0067-8.

[81] W. Jiang, N.A. Chung, J.C. May. In: Ion mobility-mass spectrometry. Encyclopedia of Analytical Chemistry: Applications, Theory and Instrumentation. New York: John Wiley \& Sons, 2013.

[82] F. Zhang, S. Guo, M. Zhang, Z. Zhang, Y. Guo. Characterizing ion mobility and collision cross section of fatty acids using electrospray ion mobility mass spectrometry. Journal of Mass Spectrometry $\mathbf{5 0}$ (2015) 906-913. https://doi.org/10.1002/ims.3600.

[83] J.C May, C.R. Goodwin, N.M. Lareau, K.L. Leaptrot, C.B. Morris, R.T. Kurulugama, A. Mordehai, C. Klein, W. Barry, E. Darland, G. Overney, K. Imatani, G.C. Stafford, J.C. Fjedsted, J.A. McLean. Conformational Ordering of Biomolecules in the gas phase: nitrogen collision cross sections measured on a prototype high resolution drift tube ion mobility-mass spectrometer. Analytical Chemistry 86 (2014) 2107-2116. https://doi.org/10.1021/ac4038448.

[84] K.A.Z. Berry, R.M. Barkley, J.J. Berry, J.A. Hankin, E. Hoyes, J.M. Brown, R.C. Murphy. Tandem mass spectrometry in combination with product ion mobility for the identification of phospholipids. Analytical Chemistry 89 (2019) 916-921. https://doi.org/10.1021/acs.analchem.6b04047.

[85] C.G. Vasilopoulou, K. Sulek, A.D. Brunner, N.S. Meitei, U. Schweiger-Hufnagel, S.W. Meyer, A. Barsch, M. Mann, F. Meier. Trapped ion mobility spectrometry and PASEF enable in-depth lipidomics from minimal sample amounts. Nature Communications 11 (2020) 331. https://doi.org/10.1038/s41467020-15613-1.

[86] Z. Zhou, J. Tu, X. Xiong, X. Shen, Z.J. Zhu. Lipid CCS: Prediction of collision cross-section values for lipids with high precision to support ion mobility-mass spectrometry-based lipidomics. Analytical Chemistry 89 (2017) 9559-9566. https://doi.org/10.1021/acs.analchem.7b02625.

[87] C. Hinz, S. Liggi, J.L. Griffin. The potential of ion mobility mass spectrometry for high-throughput and high-resolution lipidomics. Current Opinion in Chemical Biology 42 (2018) 42-50. https://doi.org/10. 1016/j.cbpa.2017.10.018.

[88] M. Groessl, S. Graf, R. Knochenmuss. High resolution ion mobility-mass spectrometry for separation and identification of isomeric lipids. Analyst 140 (2015) 6904-6911.

[89] M. Sala, M. Lisa, J.L. Campbell, M. Holcapek. Determination of triacylglycerol regioisomers using differential mobility spectrometry. Rapid Communications in Mass Spectrometry 30 (2016) 256-264. https://doi.org/10.1002/rcm.7430.

[90] A.A. Shvartsburg, G. Isaac, N. Leveque, R.D. Smith, T.O. Metz. Separation and classification of lipids using differential ion mobility spectrometry. Journal of the American Society for Mass Spectrometry 22 (2011) 1146-1155. doi: https://doi.org/10.1007/s13361-011-0114-z.

[91] F. Zandkarimi, L.M. Brown. Application of ion mobility mass spectrometry in lipidomics. Advances in Experimental Medicine and Biology 1140 (2019) 317-326. Doi: https://doi.org/10.1007/978-3-03015950-4 18.

[92] S.R. Ellis, S.H. Brown, M.I.H. Panhuis, S.J. Blanksby, T.W. Mitchell. Surface analysis of lipids by mass spectrometry: More than just imaging. Progress in Lipid Research 52 (2013) 329-353. https://doi.org/ 10.1016/i.plipres.2013.04.005.

[93] S. Shimma, Y. Sugiura, T. Hayasaka, Y. Hoshikawa, T. Noda, M. Setou. MALDI-based imaging mass spectrometry revealed abnormal distribution of phospholipids in colon cancer liver metastasis. Journal of Chromatography B 855 (2007) 98-103. https://doi.org/10.1016/i.jchromb.2007.02.037.

[94] A.C. Kendall, M.M. Koszyczarek, E.A. Jones, P.J. Hart, M. Towers, C.E.M. Griffiths, M. Morris, A. Nicolaou. Lipidomics for translational skin research: A primer for the uninitiated. Experimental Dermatology 27 (2018) 721-728. https://doi.org/10.1111/exd.13558.

[95] N. Goto-Inoue, T. Hayasaka, N. Zaima, M. Setou. Imaging mass spectrometry for lipidomics. Biochimica et Biophysica Acta - Molecular and Cell Biology Lipids 1811 (2011) 961-969. https://doi.org/10.1016/i.bbalip.2011.03.004 
[96] M.T. Bokhart, D.C. Muddiman. Infrared matrix-assisted laser desorption electrospray ionization mass spectrometry imaging analysis of biospecimens. Analyst 141 (2016) 5236-5245. Doi: https://doi.org/10.1039/c6an01189f.

[97] F. Meier, K.P. Garrard, D.C. Muddiman. Silver dopants for targeted and untargeted direct analysis of unsaturated lipids via infrared matrix-assisted laser desorption electrospray ionization (IR-MALDESI). Rapid Communication in Mass Spectrometry 28 (2014) 2461-2470. https://doi.org/10.1002/ rcm.7041.

[98] E.A. Jones, D. Simon, T. Karancsi, J. Balog, S.D. Pringle, Z. Takats. Matrix assisted rapid evaporation ionisation mass spectrometry. Analytical Chemistry 91 (2019) 9784-9791. https://doi.org/10.1021 Lacs.analchem.9b01441.

[99] F. Bolt, S.J.S. Cameron, T. Karancsi, D. Simon, R. Schaffer, T. Rickards, K. Hardiman, A. Burke, Z. Bodai, A. Perdones-Montero, M. Rebec, J. Balog, Z. Takats. Automated high-throughput identification and characterization of clinically important bacteria and fungi using rapid evaporative ionization mass spectrometry. Analytical Chemistry 88 (2016) 9419-9426. https://doi.org/10.1021/acs.analchem.$\underline{6 \mathrm{~b} 01016 .}$

[100] J. Sarsby, L. McLean, V.M. Harman, R.J. Beynon. Monitoring recombinant protein expression in bacteria by rapid evaporative ionisation mass spectrometry. Rapid Communications in Mass Spectrometry (2020) e8760. https://doi.org/10.1002/rcm.8670.

[101] K. Verplanken, S. Stead, R. Jandova, C.V. Poucke, J. Claereboudt, J.V. Bussche, S.D. Saeger, Z. Takats, J. Wauters, L. Vanhaecke. Rapid evaporative ionization mass spectrometry for high-throughput screening in food analysis: The case of boar taint. Talanta 169 (2017) 30-36. https://doi.org/10.1016/j.talanta.2017.03.056.

[102] G. Song, M. Zhang, Y. Zhang, H. Wang, S. Li, Z. Dai, Q. Shen. An in-situ method for real-time discriminating salmons and rainbow trout without sample preparation using iKnife and rapid evaporative ionization mass spectrometry based lipidomics. Journal of Agricultural and Food Chemistry 67 (2019) 4679-4688. https://doi.org/10.1021/acs.jafc.9b00751.

[103] E.S. Chernetsova, G.E. Morlock, I.A Revelsky. DART mass spectrometry and its applications in chemical analysis. Russian Chemical Reviews 80 (2011) 235-255.

[104] R.B. Cody. Observation of molecular ions and analysis of nonpolar compounds with the direct analysis in real time ion source. Analytical Chemistry 81 (2009) 1101-1107. https://doi.org $\angle 10.1021 /$ ac8022108.

[105] Z. Yang, A.B. Attygalle. Aliphatic hydrocarbon spectra by helium ionization mass spectrometry (HIMS) on a modified atmospheric-pressure source designed for electrospray ionization. Journal of The American Society for Mass Spectrometry 22 (2011) 1395-1402. https://doi.org/10.1007/s13361-0110149-1.

[106] D.A. Peake, R. Kiyonami, Y. Yokoi, Y. Fukamachi, Y. Huang. Processing of a complex lipid dataset for the NIST inter-laboratory comparison exercise for lipidomics measurements in human serum and plasma. ASMS 2015 Poster Note 64471.

(C)2021 by the authors; licensee IAPC, Zagreb, Croatia. This article is an open-access article distributed under the terms and conditions of the Creative Commons Attribution license (http://creativecommons.org/licenses/by/3.0/) (cc) E EY 